\title{
Welfare Maximizing Contest Success Functions when the Planner Cannot Commit*
}

\author{
Luis Corchón ${ }^{\dagger}$ and Matthias Dahm ${ }^{\ddagger}$
}

September 29, 2010

\begin{abstract}
We analyze how a contest organizer chooses optimally the winner when the contestants' efforts are already exerted and commitment to the use of a given contest success function is not possible. We define the notion of rationalizability in mixed-strategies to capture such a situation. Our approach allows to derive different contest success functions depending on the aims and attitudes of the decider. We derive contest success functions which are closely related to commonly used functions providing new support for them. By taking into account social welfare considerations our approach bridges the contest literature and the recent literature on political economy.

Keywords: Endogenous Contests, Contest Success Function, Mixed-Strategies.

JEL Classification: C72 (Noncooperative Games), D72 (Economic Models of Political Processes: Rent-Seeking, Elections), D74 (Conflict; Conflict Resolution; Alliances).
\end{abstract}

\section{Introduction}

In a contest players exert effort in order to win a certain prize. Contests have been used to analyze a variety of strategic situations ranging from rent-seeking and lobbying to conflict, arms races, warfare, and promotional efforts, as well as to sports. ${ }^{1}$

\footnotetext{
*This paper is dedicated to Andreu Mas-Colell who over the years provided inspiration to the authors of this paper. We wish to thank Carmen Beviá, Amihai Glazer, Carolina Manzano, Stergios Skaperdas, Bernd Theilen, Galina Zudenkova and two referees for helpful discussions and suggestions. The usual disclaimer applies. This work was carried out while Dahm was visiting the University of California, Irvine. The hospitality of this institution and the financial support through the Spanish program José Castillejo is gratefully acknowledged. The first author acknowledges financial support from the Spanish Ministerio de Educación y Ciencia, project ECO2008-02738/ECON and the second acknowledges the support of the Barcelona GSE, the Government of Catalonia and the Spanish Ministerio de Educación y Ciencia, project SEJ2007-67580-C02-01.

† Departamento de Economía. Universidad Carlos III de Madrid. Calle Madrid, 126. 28903 Getafe (Madrid). Spain. E-mail: lcorchon@eco.uc3m.es. Phone: +34 916 249617. Fax: +34 916249875.

‡ Departamento de Economía. Universitat Rovira i Virgili. Avenida de la Universitat, 1. 43204 Reus (Tarragona). Spain. E-mail: matthias.dahm@urv.cat. Phone: +34977 758903 . Fax: +34 977759810.

${ }^{1}$ For a survey see Konrad (2009).
} 
In many of these situations there is a contest organizer who awards the prize. ${ }^{2}$ For example in litigation a court decides about the winner, in lobbying, rent-seeking and rent-defending contests bureaucrats or politicians award a prize, in internal labor market tournaments jobs are allocated by the manager of an organization or in beauty contests the decision where to locate certain event is taken by a committee.

A crucial determinant for the equilibrium predictions of contests is the specification of the so-called contest success function (CSF) which relates the players' efforts and win probabilities (also interpreted as shares of the prize). ${ }^{3}$ In this paper we provide a framework in which CSFs are derived as an optimal choice of the contest administrator. ${ }^{4}$ See our remarks in the final section about the interpretation of our results when the contest is not organized by a contest administrator. The administrator is unable to commit to a CSF so she chooses the probabilities with which the prize is given in order to maximize her utility given the choices of the contestants. Assuming complete information, contestants anticipate the choice made by the contest organizer. We apply this framework by postulating different utility functions for the decider. Of course the plausibility of our results hinges on the plausibility of these utility functions. Thus we decided to use two popular utility functions namely a CES function defined on the utilities of the contenders (which generalizes the case in which the planner is utilitarian) and a (special case) of prospect theory. We also follow the recent political economy literature (see Grossman and Helpman, 2001; Persson and Tabellini, 2000) and let the decider care about social welfare - albeit in contrast to that literature in the form of a generalized utilitarian. Somewhat surprisingly, this allows to derive the three most commonly used types of CSFs: difference-form CSFs (Propositions 1, 2, 3, 5 and 6), deterministic CSFs (Proposition 4 and Example 1) and non-deterministic CSFs with an additive structure (Proposition 7).

Our approach differs from other models in which commitment is possible like the menu auction approach (see Grossman and Helpman, 1994). Dasgupta and Nti (1998) analyzed the optimal design of a contest when the contestants have identical valuations, the planner might retain the prize and the contest success function must be of the class axiomatized by Skaperdas (1996) (see equation (2) below). They find that, when the planner has a low valuation for the prize, the optimal CSF is such that the probability that a contestant obtains the prize is the ratio of her effort with respect to total effort. A closely related paper is Epstein and Nitzan (2006) which with respect to commitment can be seen as the opposite benchmark to our paper. In Epstein and Nitzan the contest organizer decides first whether to have a contest at all, and if a contest takes place, she chooses the CSF among the elements of a fixed set of

\footnotetext{
${ }^{2}$ We use the terms contest administrator, contest organizer, decider and planner interchangeably. Participants in the contest are called contestants, rent-seekers or contenders.

${ }^{3} \mathrm{~A}$ prominent example for the interpretation as shares is Wärneryd (1998). He analyzes a contest among jurisdictions for shares of the GNP and compares different types of jurisdictional organization.

${ }^{4}$ Other alternative foundations for CSFs are provided by Blavatskyy (2010), Münster (2009) and Rai and Sarin (2009) who offer axiomatic characterizations of contest success functions following the seminal paper by Skaperdas (1996). The earlier work on foundations for contest success functions has been extensively reviewed by Konrad (2009).
} 
CSFs maximizing ex-ante utility. In doing so, the organizer anticipates the equilibrium efforts of contestants and needs to be able to commit to employ a given CSF once efforts are exerted. In contrast, in our approach the organizer decides about the winner once efforts are exerted. ${ }^{5}$ There are further approaches motivating or endogeneizing the CSF and some of them imply that it is not needed to assume commitment. In one recent approach contestants might be uncertain about a characteristic of the organizer and as a result view the determination of the winner as probabilistic although the organizer chooses in a deterministic way (Corchón and Dahm, 2010; Skaperdas and Vaidya, 2010). In another, efforts are affected by exogenous shocks so that the performance of contestants is different from efforts which generates randomness from the point of view of contestants (Jia, 2008).

Our approach cannot be construed as a criticism of the commitment assumption. The commitment case is an important benchmark case. It is, however, not always clear how the decider can be trusted to maintain her word. In other cases the planner may prefer a policy of wait and see instead of announcing a certain CSF. Therefore, it is important to know what happens when, after contestants have exerted efforts, the contest administrator is no longer constrained by her word and could choose the winner in the way she pleases. Thus our paper can be viewed as a check to the properties of CSFs in case commitment becomes unlikely. Our main conclusion is that well-known CSFs arise under natural specifications of the preferences of the contest organizer. Thus our approach could be considered as a back up to the use of well-known CSFs.

\section{Preliminaries}

A contest administrator conducts a contest among $n$ contestants denoted by $i \in N=\{1, \ldots, n\}$. Each contestant has a valuation for the prize, denoted by $V_{i} \in \mathbb{R}_{+}$, and exerts effort $G_{i} \in \mathbb{R}_{+}$ in order to affect the probability of winning the prize which is given by the CSF.

Formally, a contest success function $\boldsymbol{p}(\boldsymbol{G})=\left(p_{1}(\boldsymbol{G}), p_{2}(\boldsymbol{G}) \ldots, p_{n}(\boldsymbol{G})\right)$ associates, to each vector of efforts $\boldsymbol{G}$, a lottery specifying for each agent a probability $p_{i}$ of getting the prize. That is, $p_{i}=p_{i}(\boldsymbol{G})$ is such that, for each contestant $i \in N, p_{i}(\boldsymbol{G}) \geq 0$, and $\sum_{i=1}^{n} p_{i}(\boldsymbol{G})=1$.

We say that a CSF is imperfectly discriminating if $G_{i}>0$ implies that $p_{i}(\boldsymbol{G})>0 .{ }^{6}$ An example for such a function is the most commonly used CSF introduced by Tullock (1980) which is given by

$$
p_{i}=\frac{G_{i}^{R}}{\sum_{j=1}^{n} G_{j}^{R}}, \text { for } i=1, \ldots, n,
$$

\footnotetext{
${ }^{5}$ There are further differences to our paper. In our approach the contest organizer is completely unconstrained in her choice of the contest success function, rather than choosing among the elements of a fixed set of contest success functions. Also, our approach is not restricted to the case of two contestants.

${ }^{6}$ This is essentially axiom 1 in Skaperdas (1996). The name of this property refers to the fact that a contest can be interpreted as an auction where the prize is auctioned among the agents and efforts are bids. In standard auctions the highest bid obtains the prize with probability one. Here, any positive bid entitles the bidder with a positive probability to obtain the object, so it is as if the bidding mechanism did not discriminate perfectly among bids.
} 
where $R$ is a positive parameter. A generalization of this form is

$$
p_{i}=\frac{f_{i}\left(G_{i}\right)}{\sum_{j=1}^{n} f_{j}\left(G_{j}\right)}, \text { for } i=1, \ldots, n,
$$

where the $f_{i}(\cdot)$ are positive increasing functions of its argument. For the case in which $f_{i}(\cdot)=f(\cdot)$ for $i=1, \ldots, n,(2)$ has been axiomatized by Skaperdas (1996). Another class of CSFs are difference-forms. While the difference-form in Hirshleifer (1989) is a special case of (2), the ones in Baik (1998) and Che and Gale (2000) are not. The linear difference-form CSF in Che and Gale (2000) is defined as

$$
p_{i}=\max \left\{\min \left\{\frac{1}{2}+s\left(G_{i}-G_{j}\right), 1\right\}, 0\right\} \text { for } i=1,2 \text { and } j \neq i,
$$

where $s$ is a positive scalar. Notice that the linear difference-form is not imperfectly discriminating. Another example for a not imperfectly discriminating CSF is a function that assigns probability one to the contestant exerting the highest effort, like in an all-pay auction.

Contestants are risk-neutral. When the contest is all-pay (effort is irreversible so that all contestants pay their bid), the expected utility of a contestant is

$$
u_{i}\left(p_{i}, \boldsymbol{G}\right)=p_{i}(\boldsymbol{G}) V_{i}-G_{i} .
$$

In contrast when the contest is winner-pay (effort is like a promise so that only the winner pays his bid)

$$
u_{i}\left(p_{i}, \boldsymbol{G}\right)=p_{i}(\boldsymbol{G})\left(V_{i}-G_{i}\right) .
$$

While contests are usually analyzed as all-pay, winner-pay contests have been analyzed in Skaperdas and Gan (1995), Wärneryd (2000) and Yates (2010). There is also a large literature on the first-price (sealed bid) auction which constitutes the extreme case of a winner-pay contest in which the highest bidder wins with probability one.

The timing is as follows. In an all-pay contest contenders exert effort in the first stage (simultaneously), while the administrator assigns win probabilities or shares of the prize in the second stage. In a winner-pay contest contenders promise effort in the first stage (simultaneously) but effort is not exerted yet. In the second stage the organizer determines the outcome of the contest. In the third stage the winner exerts the efforts promised. ${ }^{7}$ When the contest is all-pay and contestants play mixed strategies, in stage 1 contestants anticipate the CSF and choose a mixed strategy. Then the actual effort levels are realized and the organizer observes them. In stage two the organizer chooses (given the realization of efforts) the CSF which is the one contestants have anticipated in stage 1 . The timing is similar when the contest is winner-pay and contestants play mixed strategies. The only change is that the organizer chooses the CSF based on promises of effort levels, chosen by the contestants as the realization of the mixed-strategies. Again, in the third stage the winner exerts the effort promised.

\footnotetext{
${ }^{7}$ Our approach also works if in the first stage contestants exert effort sequentially (in the case of the all-pay contest) or promise effort sequentially (in the case of the winner-pay contest). We assume that in a winner-pay contest the promise of effort in stage one will be fulfilled in stage three. A reason for this could be that the prize will only be delivered when effort has been exerted.
} 


\section{Rationalizability in Mixed-Strategies}

In this section we formalize the idea that the contest organizer's choice in the second stage is optimal from her view point. Suppose that, after effort has been exerted or promised in the first stage, the decider assigns win probabilities or shares of the prize maximizing her objective function. Since there is no uncertainty and contestants know the organizer and her incentives, rent-seekers are able to anticipate in the first stage which CSF will be chosen.

We capture the idea that a particular CSF, which is used when contestants make their effort choices, is also optimal from the point of view of the contest organizer with the following definition. Denote by $S^{n}$ the $n-1$ dimensional simplex.

Definition 1 The contest success function $\boldsymbol{p}(\boldsymbol{G})=\left(p_{1}(\boldsymbol{G}), p_{2}(\boldsymbol{G}) \ldots, p_{n}(\boldsymbol{G})\right)$ is rationalizable in mixed-strategies if there is a function $W(\boldsymbol{p}, \boldsymbol{G})$ such that for all $\boldsymbol{G} \in \mathbb{R}^{n}$,

$$
\boldsymbol{p}(\boldsymbol{G})=\operatorname{argmax} W(\boldsymbol{p}, \boldsymbol{G}), \boldsymbol{p} \in S^{n} .
$$

Here the term mixed-strategies refers to the fact that technically a CSF is a probability distribution even though, as we have already mentioned, it might be interpreted as shares of the prize. In our approach, the contest success function is the best reply of a contest organizer with payoff function $W(\boldsymbol{p}, \boldsymbol{G})$. As we will see in the sequel, the payoff function allows to take into account a variety of objectives and attitudes of the decider. The use of mixed-strategies here might be motivated analogously to the classical argument in favor of mixed-strategies, namely that mixed-strategies produce unpredictable choices that cannot be exploited by an opponent. This might translate to enabling the organizer to avoid that contestants forecast perfectly her choice, which might reduce incentives to exert effort. There are, however, differences between our approach and the way mixed-strategies are usually employed. Usually a player chooses a mixedstrategy when she is indifferent between the pure strategies involved. In contrast, we will be able to derive mixed-strategies which are strictly preferred to any other pure or mixed-strategy. This is because our planners are not expected utility maximizers, even though they are utility maximizers (see also Cheng and Zhu, 1995, who avoid indifferences among mixed-strategies by using quadratic utility).

\section{Generalized CES Utilitarian Planner}

As commonly assumed in the recent political economy literature (see Grossman and Helpman, 2001; Persson and Tabellini, 2000) let the decider's objective function depend on expected social welfare. ${ }^{8}$ Consider a generalized CES utilitarian planner whose payoff function is a constant

\footnotetext{
${ }^{8}$ In that literature the objective function is a weighted average of social welfare and lobbying efforts. We are grateful to a referee for pointing out that none of our results would change if we assumed that the planner maximizes a convex combination of the objective functions specified below and total effort.
} 
elasticity of substitution function

$$
W(\boldsymbol{p}, \boldsymbol{G})=\left\{\begin{array}{cc}
\left(\sum_{i=1}^{n}\left(u_{i}\left(p_{i}, \boldsymbol{G}\right)\right)^{1-r}\right)^{1 /(1-r)} & \text { if } \quad r \neq 1 \\
\sum_{i=1}^{n} \ln \left(u_{i}\left(p_{i}, \boldsymbol{G}\right)\right) & \text { if } \quad r=1
\end{array} .\right.
$$

This function is well known in the literature (see the discussion in Bossert and Weymark, 2004, pp. 1161-1164). The positive parameter $r$ represents the degree of inequality aversion of the planner. If the CSF specifies win probabilities, inequality can be interpreted as referring to expected utility. The utilitarian case corresponds to $r=0$. When $r=1$, the Bernoulli-Nash case obtains. When $r$ goes to infinity, the Rawlsian case arises and the concern is with the least well-off only.

We consider an all-pay contest. ${ }^{9}$ The planner maximizes $W(\boldsymbol{p}, \boldsymbol{G})$ as defined in (4) with respect to $\boldsymbol{p}$. In most of the results of this section we assume the existence of an interior solution to this program. We discuss this assumption after Corollary 2 and provide a sufficient condition for the existence of such solutions. It is instructive to start with the Bernoulli-Nash case in which $r=1$.

Proposition 1 Suppose the contest is all-pay and maximizing (4) with respect to $\boldsymbol{p}$ has an interior solution. The contest success function

$$
p_{i}=\frac{1-\sum_{j=1}^{n}\left(G_{j} / V_{j}\right)}{n}+G_{i} / V_{i} \text { for } i=1, \ldots, n
$$

is the unique that can be rationalized in mixed-strategies by a function fulfilling (4) with $r=1$.

Proof. Notice first that the objective function is strictly concave, as the Hessian matrix is a diagonal matrix which has as its $i$ th element $-\left(V_{i}\right)^{2} /\left(p_{i} V_{i}-G_{i}\right)^{2}$. Since the solution is interior, the first order conditions imply

$$
\left(p_{i} V_{i}-G_{i}\right) V_{j}=\left(p_{j} V_{j}-G_{j}\right) V_{i}, \text { for } i, j=1, \ldots, n \text {. }
$$

Rearranging we obtain

$$
p_{i}=\frac{p_{n} V_{n}-G_{n}}{V_{n}}+\frac{G_{i}}{V_{i}} \text { for } i=1, \ldots, n .
$$

Adding up over all contestants yields

$$
p_{n}=\frac{1-\sum_{j=1}^{n}\left(G_{j} / V_{j}\right)}{n}+G_{n} / V_{n}
$$

and replacing this in the previous equation we obtain (5).

Notice that the expression in (5) is a generalized difference-form. To see this consider the following corollary.

\footnotetext{
${ }^{9}$ We omit the discussion of winner-pay contests as the mathematical structure of the problem is closely related to Proposition 7. See the discussion after that proposition.
} 
Corollary 1 Suppose the contest is all-pay and maximizing (4) with respect to $\boldsymbol{p}$ has an interior solution.

1. If there is a common value $V$, the contest success function

$$
p_{i}=\frac{1}{n}+\frac{1}{n V}\left((n-1) G_{i}-\sum_{j \neq i} G_{j}\right) \text { for } i=1, \ldots, n
$$

is the unique that can be rationalized in mixed-strategies by a function fulfilling (4) with $r=1$.

2. If $n=2$, the contest success function

$$
p_{i}=\frac{1}{2}+\frac{1}{2}\left(\frac{G_{i}}{V_{i}}-\frac{G_{j}}{V_{j}}\right) \text { for } i=1,2 \text { and } j \neq i
$$

is the unique that can be rationalized in mixed-strategies by a function fulfilling (4) with $r=1$.

Notice that the CSFs in (6) and (7) are very related to the linear difference-form contest in Che and Gale (2000) given in (3). When there are two contestants and when $p_{i}>0$ for $i=1,2$, then (5) coincides with (3) if there is a common value $V$ and $V=1 /(2 s)$. This threshold $V=1 /(2 s)$ has the interesting interpretation that a contestant can only guarantee success by exerting at least this amount more than the opponent.

As Baik (1998) and Che and Gale (2000) have shown, in many difference-form contests there are problems concerning the existence of pure strategy equilibria. But, as we already discussed, our approach can also work when contestants play a mixed-strategy.

We consider now the case in which $r \neq 1$ and $r \in(0, \infty)$.

Proposition 2 Suppose the contest is all-pay and maximizing (4) with respect to $\boldsymbol{p}$ has an interior solution. The contest success function

$$
p_{i}=\frac{1-\sum_{j=1}^{n}\left(G_{j} / V_{j}\right)}{\sum_{j=1}^{n}\left(V_{j} / V_{i}\right)^{\frac{1-r}{r}}}+G_{i} / V_{i} \text { for } i=1, \ldots, n
$$

is the unique that can be rationalized in mixed-strategies by a function fulfilling (4) with $r \neq 1$ and $r \in(0, \infty)$.

Proof. Suppose $r \neq 1$ and $r \in(0, \infty)$ and consider

$$
W^{\prime}(\boldsymbol{p}, \boldsymbol{G})=\sum_{i=1}^{n}\left(p_{i} V_{i}-G_{i}\right)^{1-r} .
$$

Notice that for $r<1, W(\boldsymbol{p}, \boldsymbol{G})$ is an increasing transformation of $W^{\prime}(\boldsymbol{p}, \boldsymbol{G})$, while for $r>1$, $W(\boldsymbol{p}, \boldsymbol{G})$ is a decreasing transformation of $W^{\prime}(\boldsymbol{p}, \boldsymbol{G})$. The Hessian matrix of $W^{\prime}(\boldsymbol{p}, \boldsymbol{G})$ is a 
diagonal matrix which has as its $i$ th element $-r(1-r)\left(V_{i}\right)^{2}\left(p_{i} V_{i}-G_{i}\right)^{-r-1} \cdot W^{\prime}(\boldsymbol{p}, \boldsymbol{G})$ is, hence, strictly concave for $r<1$ and strictly convex for $r>1$. $W^{\prime}(\boldsymbol{p}, \boldsymbol{G})$ has thus a unique maximizer in the first case and a unique minimizer in the latter case. Both correspond to the unique maximizer of $W(\boldsymbol{p}, \boldsymbol{G})$.

Let us maximize $W^{\prime}(\boldsymbol{p}, \boldsymbol{G})$ as defined above subject to $p \in S^{n}$, where $S^{n}$ is the $n-1$ dimensional simplex. Since the solution is interior, the first order conditions imply

$$
\left(p_{i} V_{i}-G_{i}\right)^{r} V_{j}=\left(p_{j} V_{j}-G_{j}\right)^{r} V_{i}, \text { for } i, j=1, \ldots, n .
$$

Rearranging we obtain

$$
p_{i}=\frac{p_{n} V_{n}-G_{n}}{\left(V_{n}\right)^{1 / r}}\left(V_{i}\right)^{\frac{1-r}{r}}+\frac{G_{i}}{V_{i}} \text { for } i=1, \ldots, n .
$$

Adding up over all contestants yields

$$
p_{n}=\frac{1-\sum_{j=1}^{n}\left(G_{j} / V_{j}\right)}{\sum_{j=1}^{n}\left(V_{j}\right)^{\frac{1-r}{r}}}\left(V_{n}\right)^{\frac{1-r}{r}}+G_{n} / V_{n}
$$

and replacing this in the previous equation we obtain (8).

Notice that when $r$ goes to one, (8) becomes (5), while when there is a common value (8) becomes (6). Consider now the Rawlsian case in which $r$ goes to infinity.

Proposition 3 Suppose the contest is all-pay and maximizing (4) with respect to $\boldsymbol{p}$ has an interior solution. The contest success function

$$
p_{i}=\frac{1-\sum_{j=1}^{n}\left(G_{j} / V_{j}\right)}{\sum_{j=1}^{n} V_{i} / V_{j}}+G_{i} / V_{i} \text { for } i=1, \ldots, n
$$

is the unique that can be rationalized in mixed-strategies by a function fulfilling (4) with $r=\infty$.

Proof. For $r$ equal to infinity the objective function (4) becomes

$$
W(\boldsymbol{p}, \boldsymbol{G})=\min \left\{p_{1} V_{1}-G_{1}, \ldots, p_{n} V_{n}-G_{n}\right\} .
$$

If the solution is interior, it must hold that

$$
p_{i} V_{i}-G_{i}=p_{j} V_{j}-G_{j}, \text { for } i, j=1, \ldots, n .
$$

This implies that

$$
p_{i}=\frac{p_{n} V_{n}-G_{n}}{V_{i}}+\frac{G_{i}}{V_{i}} \text { for } i=1, \ldots, n .
$$

Adding up over all contestants yields

$$
p_{n}=\frac{1-\sum_{j=1}^{n}\left(G_{j} / V_{j}\right)}{\sum_{j=1}^{n} 1 / V_{j}}\left(V_{n}\right)^{-1}+G_{n} / V_{n}
$$

and replacing this in the previous equation we obtain (9).

Notice that when $r$ goes to infinity, (8) becomes (9). Note also that when there is a common value all cases considered so far yield the same CSF. 
Corollary 2 Suppose the contest is all-pay, maximizing (4) with respect to $\boldsymbol{p}$ has an interior solution and there is a common value. The contest success function (6) is the unique that can be rationalized in mixed-strategies by a function fulfilling (4) with $r>0$.

We may also consider an individual rationality constraint in the planner's problem adding the restrictions that $u_{i} \geq 0, i=1,2, \ldots, n$. Notice that in the cases considered in Propositions 1 , 2 and 3 as long as $\sum_{j=1}^{n} G_{j} / V_{j} \leq 1$ it can be easily shown that there is a subset of the simplex from which win probabilities can be chosen such that the individual rationality constraint is satisfied. Note also that in the case of a common value the assumption that the sum of individual efforts weighted by the valuation must be smaller than one says that the rent is not completely dissipated.

The assumption that $\sum_{j=1}^{n} G_{j} / V_{j} \leq 1$ together with $G_{i} / V_{i}>0, i=1,2, \ldots, n$ is also sufficient to guarantee that maximizing $(4$, for $r \neq 0)$ with respect to $\boldsymbol{p}$ has an interior solution. This shows that our interiority assumption is satisfied under certain conditions. If the interiority assumption holds for at least two contestants, Propositions 1, 2 and 3 are still valid interpreting the set of players as the set of contestants for which this assumption holds.

Finally let us consider the case of $r=0$ in which the planner is utilitarian. In this case, in general, there will be no interior solution and the assignment of win probabilities depends only on valuations. More precisely, the planner prefers that the contestant with the highest valuation wins the prize. In the common value case the objective function of the planner becomes insensitive to whom wins the contest.

Proposition 4 Suppose the contest is all-pay and $W(\boldsymbol{p}, \boldsymbol{G})$ follows (4) with $r=0$. The contest success function is such that the contestant with the highest valuation wins the prize: If $p_{i}>0$, then $V_{i}=\max \left\{V_{1}, V_{2}, \ldots, V_{n}\right\}$.

\section{Extensions}

We now extend the approach of the previous section in several dimensions. It turns out that under all these extensions the basic structure of the resulting CSFs is similar to those derived before.

\subsection{Alternative Objective Functions for the Planner}

In the previous section we derived the linear difference-form CSF as the only outcome of the optimization performed by a planner whose payoff function is a constant elasticity of substitution function defined over participants' expected utilities. A natural question is to ask if by changing the form of the utility function of the planner but keeping the assumption that it is defined over participants' expected utilities, other CSFs can be rationalizable. In this section we give a partial answer to this question for the case of two agents. This case is important because many situations of pure conflict involve two players alone. However, before doing so we analyze the implications of an alternative formalization of inequality aversion. 


\subsubsection{Constant Absolute Inequality Aversion}

So far we have interpreted the parameter $r$ in the difference-form CSFs derived from the generalized utilitarian planner as the degree of inequality aversion. We show now that similar difference-form CSFs can be obtained assuming that the planner cares about social welfare in a different way. This shows robustness of our results and allows to interpret a parameter of the arising CSFs as a coefficient of inequality aversion. Following Ray and Ueda (1996), let the planner's payoff function be given by

$$
W(\boldsymbol{p}, \boldsymbol{G})=-\frac{1}{\eta} \sum_{i=1}^{n}\left\{\exp \left(-\eta u_{i}\left(p_{i}, \boldsymbol{G}\right)\right)-1\right\}, \eta \in(0, \infty) .
$$

The parameter $\eta$ is the coefficient of absolute risk aversion. ${ }^{10}$ Higher values of $\eta$ are associated with a more egalitarian function. The utilitarian case corresponds to $\eta=0$, while the Rawlsian case obtains when $\eta=\infty$.

Proposition 5 Suppose the contest is all-pay and maximizing (11) with respect to $\boldsymbol{p}$ has an interior solution. The contest success function

$$
p_{i}=\frac{1-\sum_{j=1}^{n}\left(G_{j} / V_{j}\right)}{\sum_{j=1}^{n} V_{i} / V_{j}}+G_{i} / V_{i}-\frac{\sum_{j=1}^{n} \frac{\ln \left(V_{j} / V_{i}\right)}{\eta V_{j}}}{\sum_{j=1}^{n} V_{i} / V_{j}} \text { for } i=1, \ldots, n
$$

is the unique that can be rationalized in mixed-strategies by a function fulfilling (11).

Proof. Notice first that the objective function is strictly concave, as the Hessian matrix is a diagonal matrix which has as its $i i$ th element $-\eta\left(V_{i}\right)^{2} \exp \left(-\eta u_{i}\left(p_{i}, \boldsymbol{G}\right)\right)$. Since the solution is interior, the first order conditions imply

$$
\exp \left(-\eta u_{i}\left(p_{i}, \boldsymbol{G}\right)\right) V_{i}=\exp \left(-\eta u_{j}\left(p_{j}, \boldsymbol{G}\right)\right) V_{j}, \text { for } i, j=1, \ldots, n \text {. }
$$

Taking logarithms and rearranging we obtain

$$
p_{i}=\frac{G_{i}-G_{n}}{V_{i}}+\frac{p_{n} V_{n}}{V_{i}}+\frac{\ln \left(V_{i} / V_{n}\right)}{\eta V_{i}} \text { for } i=1, \ldots, n .
$$

Adding up over all contestants yields

$$
p_{n}=\frac{1-\sum_{j=1}^{n}\left(G_{j} / V_{j}\right)-\sum_{j=1}^{n} \frac{\ln \left(V_{j} / V_{n}\right)}{\eta V_{j}}}{\sum_{j=1}^{n} V_{n} / V_{j}}+G_{n} / V_{n}
$$

and replacing this in the previous equation we obtain (12).

Note that the CSF in (12) adds to the one in (9) the last term which biases the CSF in favor of contestants with higher valuations. The weight of the bias decreases in $\eta$. In the extreme,

\footnotetext{
${ }^{10}$ Notice that the results in the previous section can be interpreted as arising from constant relative inequality aversion of the planner.
} 
when $\eta$ goes to infinity and the Rawlsian case is obtained, (12) coincides with (9). As in the case of the generalized utilitarian planner with any $r>0$, when there is a common value the CSF in (6) is the only one that can be rationalized. The latter is also true when there is a common value and the planner's utility function is generalized to the class of additively separable functions, which includes (11).

Proposition 6 Suppose the contest is all-pay, there is a common value and the planner's utility function is additively separable, written $W(\boldsymbol{p}, \boldsymbol{G})=\sum_{i=1}^{n} \Psi\left(p_{i} V-G_{i}\right)$ with $\Psi(\cdot)$ strictly concave. Under interiority of the solution, the contest success function (6) is the unique that can be rationalized in mixed strategies.

Proof. The first order conditions of social welfare maximization imply that

$$
\frac{\partial \Psi\left(p_{i} V-G_{i}\right)}{\partial u_{i}}=\frac{\partial \Psi\left(p_{j} V-G_{j}\right)}{\partial u_{j}}, \text { for all } i, j .
$$

The strict concavity of $\Psi(\cdot)$ implies that $p_{i} V-G_{i}=p_{j} V-G_{j}$ for all $i$ and $j$. Thus by the same reasoning used in the proof of, say, Proposition 1, this implies the linear CSF in (6).

\subsubsection{Generalized Utilitarian Planner}

The analysis in the previous subsection calls for a more general view of the issue of rationalizability. Let $\boldsymbol{V}$ be a list of valuations. A planner is generalized utilitarian if her utility function (called generalized utilitarian $)$ is $W\left(u_{1}, u_{2}, \ldots, u_{n}\right)$ or equivalently $W\left(u_{1}\left(\boldsymbol{G}, V_{1}\right), u_{2}\left(\boldsymbol{G}, V_{2}\right), \ldots, u_{n}\left(\boldsymbol{G}, V_{n}\right)\right)$. We adapt the general definition of rationalizability to our case.

Definition $2 A$ contest success function $\boldsymbol{p}(\boldsymbol{G}, \boldsymbol{V})=\left(p_{1}(\boldsymbol{G}, \boldsymbol{V}), p_{2}(\boldsymbol{G}, \boldsymbol{V}), \ldots, p_{n}(\boldsymbol{G}, \boldsymbol{V})\right)$ is rationalizable in mixed-strategies by a generalized utilitarian planner if it maximizes a generalized utilitarian function for given $\boldsymbol{G}$ and $\boldsymbol{V}$.

It is implicit in the definition above that the generalized utilitarian function must yield the CSF for all possible values of $\boldsymbol{G}$ and $\boldsymbol{V}$ namely for all elements in $\mathbb{R}_{+}^{2 n}$. The question is what kind of CSFs might arise in this more general framework. We have a partial answer in the case of two agents when all functions are continuously differentiable. Let

$$
M\left(u_{1}, u_{2}\right) \equiv \frac{\frac{\partial W\left(u_{1}, u_{2}\right)}{\partial u_{1}}}{\frac{\partial W\left(u_{1}, u_{2}\right)}{\partial u_{2}}}
$$

be the marginal rate of substitution between the expected utility of agents 1 and 2 . We will assume that this magnitude is always well-defined, i.e. $\frac{\partial W\left(u_{1}, u_{2}\right)}{\partial u_{2}} \neq 0$.

Lemma 1 Let $n=2$ and $W(\cdot)$ be twice continuously differentiable. If a CSF is continuously differentiable and rationalizable in mixed-strategies by a generalized utilitarian planner,

$$
\frac{d p_{1}(\boldsymbol{G}, \boldsymbol{V})}{d G_{1}} \frac{\partial M\left(u_{1}, u_{2}\right)}{\partial u_{2}}=\frac{d p_{1}(\boldsymbol{G}, \boldsymbol{V})}{d G_{2}} \frac{\partial M\left(u_{1}, u_{2}\right)}{\partial u_{1}} .
$$


Proof. First notice that $p_{2}(\boldsymbol{G}, \boldsymbol{V})=1-p_{1}(\boldsymbol{G}, \boldsymbol{V})$. Consider the first order conditions of utility maximization of the generalized utilitarian planner

$$
\max W\left(p_{1} V_{1}-G_{1},\left(1-p_{1}\right) V_{2}-G_{2}\right)
$$

which are

$$
\frac{\frac{\partial W\left(u_{1}, u_{2}\right)}{\partial u_{1}}}{\frac{\partial W\left(u_{1}, u_{2}\right)}{\partial u_{2}}}=\frac{V_{2}}{V_{1}} .
$$

Using the notation introduced before, (13) can be written as

$$
M\left(p_{1} V_{1}-G_{1},\left(1-p_{1}\right) V_{2}-G_{2}\right)=\frac{V_{2}}{V_{1}}
$$

Notice that the CSF $p_{1}\left(G_{1}, G_{2}\right)$ is a solution of (14). Differentiating (14) with respect to $G_{1}$ and $G_{2}$ we get

$$
\begin{aligned}
\frac{d p_{1}(\boldsymbol{G}, \boldsymbol{V})}{d G_{1}} & =\frac{\frac{\partial M\left(u_{1}, u_{2}\right)}{\partial u_{1}}}{\frac{\partial M\left(u_{1}, u_{2}\right)}{\partial u_{1}} V_{1}-\frac{\partial M\left(u_{1}, u_{2}\right)}{\partial u_{2}} V_{2}}, \\
\frac{d p_{1}(\boldsymbol{G}, \boldsymbol{V})}{d G_{2}} & =\frac{\frac{\partial M\left(u_{1}, u_{2}\right)}{\partial u_{2}}}{\frac{\partial M\left(u_{1}, u_{2}\right)}{\partial u_{1}} V_{1}-\frac{\partial M\left(u_{1}, u_{2}\right)}{\partial u_{2}} V_{2}},
\end{aligned}
$$

and the result follows.

Notice that in the case of a generalized CES utilitarian planner specified in (4), $\partial M / \partial u_{1}=$ $-\left(V_{2} / V_{1}\right)^{1 / r} \partial M / \partial u_{2}$ so $d p_{1} / d G_{1}=1 / V_{1} V_{2}^{(1-r) / r} /\left(V_{1}^{(1-r) / r}+V_{2}^{(1-r) / r}\right)$, as shown in the previous section. When the planner has constant absolute inequality aversion given by (11), then $\partial M / \partial u_{1}=-\partial M / \partial u_{2}$ and as before $d p_{1} / d G_{1}=1 /\left(V_{1}+V_{2}\right)$. In both cases $V_{1}=V_{2}$ implies $d p_{1} / d G_{1}=1 /\left(2 V_{1}\right)$.

Now we state a consequence of Lemma 1. A condition sometimes imposed on CSFs is homogeneity of degree zero. This condition reflects the fact that the units in which efforts are measured should have no impact on the result of the contest. Skaperdas (1996) used this condition in the axiomatization of the Tullock CSF. We also assume without explicitly stating that $d p_{1} / d G_{1}>0$ for all $G_{1}, d p_{1} / d G_{2}<0$ for all $G_{2}$ and that $W(\cdot)$ is symmetric in the following sense: when $u_{i}=u_{j}$ then $\partial W / \partial u_{i}=\partial W / \partial u_{j}, \partial^{2} W /\left(\partial u_{i} \partial u_{i}\right)=\partial^{2} W /\left(\partial u_{j} \partial u_{j}\right)$ and $\partial^{2} W /\left(\partial u_{i} \partial u_{j}\right)=\partial^{2} W /\left(\partial u_{j} \partial u_{i}\right)$.

Corollary 3 Under the conditions of Lemma 1 no CSF can be rationalized in mixed strategies by a generalized utilitarian planner if it is either (i) homogeneous of degree zero or (ii) homogeneous of any degree $\alpha \neq 0$ and $p_{1}\left(G_{1}, G_{1}, V_{1}, V_{1}\right)=1 / 2$.

Proof. The corollary follows from the fact that homogeneity of degree zero implies that

$$
\frac{\frac{d p_{1}(\boldsymbol{G}, \boldsymbol{V})}{d G_{1}}}{\frac{d p_{1}(\boldsymbol{G}, \boldsymbol{V})}{d G_{2}}}\left[1-\frac{\alpha p_{1}(\boldsymbol{G}, \boldsymbol{V})}{\frac{d p_{1}(\boldsymbol{G}, \boldsymbol{V})}{d G_{1}} G_{1}}\right]=\frac{-G_{2}}{G_{1}} .
$$


Equation (17) and Lemma 1 imply that

$$
\frac{\frac{\partial M\left(u_{1}, u_{2}\right)}{\partial u_{1}}}{\frac{\partial M\left(u_{1}, u_{2}\right)}{\partial u_{2}}}\left[1-\frac{\alpha p_{1}(\boldsymbol{G}, \boldsymbol{V})}{\frac{d p_{1}(\boldsymbol{G}, \boldsymbol{V})}{d G_{1}} G_{1}}\right]=\frac{-G_{2}}{G_{1}}
$$

for all $G_{1}, G_{2}, u_{1}, u_{2}$. When $\alpha=0$, the last equation requires that

$$
\frac{\frac{\partial M\left(u_{1}, u_{2}\right)}{\partial u_{1}}}{\frac{\partial M\left(u_{1}, u_{2}\right)}{\partial u_{2}}}=\frac{-G_{2}}{G_{1}}
$$

holds for all $G_{1}, G_{2}, u_{1}, u_{2}$. Fix $G_{1}$ and $G_{2}$. By varying $V_{1}$ and $V_{2}$ all the positive values of $u_{1}$ and $u_{2}$ can be reached. Thus, the left hand side of (19) must be constant on $u_{1}$ and $u_{2}$ when these variables are positive. But then equation (19) cannot hold for all values of $G_{1}$ and $G_{2}$.

Assume $\alpha>0$. Straightforward computation yields that

$$
\frac{\frac{\partial M\left(u_{1}, u_{2}\right)}{\partial u_{1}}}{\frac{\partial M\left(u_{1}, u_{2}\right)}{\partial u_{2}}}=-\frac{\frac{\partial^{2} W}{\partial u_{1} \partial u_{1}} \frac{\partial W}{\partial u_{2}}-\frac{\partial^{2} W}{\partial u_{2} \partial u_{1}} \frac{\partial W}{\partial u_{1}}}{\frac{\partial^{2} W}{\partial u_{2} \partial u_{2}} \frac{\partial W}{\partial u_{1}}-\frac{\partial^{2} W}{\partial u_{1} \partial u_{2}} \frac{\partial W}{\partial u_{2}}} .
$$

Fix any $G_{1}=G_{2}$ and $V_{1}=V_{2}$. Suppose $p_{1}\left(G_{1}, G_{1}, V_{1}, V_{1}\right)=1 / 2$. Thus $u_{1}=u_{2}$ and by symmetry of $W(\cdot)$ we have $\partial M / \partial u_{1}=-\partial M / \partial u_{2}$. This implies that equation (18) can be rewritten and

$$
\frac{\alpha}{2 G_{1}}\left[\frac{d p_{1}(\boldsymbol{G}, \boldsymbol{V})}{d G_{1}}\right]^{-1}=0
$$

must hold for all $G_{1}>0$ contradicting that $\alpha>0$.

\subsection{More General Cost Functions of Effort}

The difference-form CSFs derived so far are linear in the effort of contestants. Our approach can be generalized in order to yield CSFs which are non-linear in effort. Suppose expected utility is given by

$$
u_{i}\left(p_{i}, \mathbf{G}\right)=p_{i}(\boldsymbol{G}) V_{i}-C_{i}\left(G_{i}\right),
$$

where a contestant's cost of effort are given by $C_{i}\left(G_{i}\right)$. It is straightforward to verify that e.g. the CSF for the Bernoulli-Nash case, given in (5), becomes

$$
p_{i}=\frac{1-\sum_{j=1}^{n}\left(C_{j}\left(G_{j}\right) / V_{j}\right)}{n}+C_{i}\left(G_{i}\right) / V_{i} \text { for } i=1, \ldots, n,
$$

and that the other cases can be extended similarly.

\subsection{More General Prize Structure}

Following the literature on cooperative production suppose that the value of the prize depends on effort. For example, suppose that $V_{i}=\hat{V}_{i}+a \sum_{j=1}^{n} f_{j}\left(G_{j}\right)$, with $a \geq 0$ and $\hat{V}_{i}>0$. Here a contestant values the prize for two reasons. First, there is an intrinsic component $\hat{V}_{i}$. Second, 
there is another component reflecting aggregate merit. The parameter $a$ is the marginal rate of substitution between aggregate merit and intrinsic value of the prize. The case where merits do not add value to the prize corresponds to $a=0$. Since $V_{i}$ is independent of $\boldsymbol{p}$, this generalization does not affect the planner's problem and the same CSFs arise. Notice, however, that as in the extension in the previous subsection the CSFs derived are non-linear in effort. ${ }^{11}$

A final remark is in order. It is well-known that in difference-form contests pure strategy equilibria have very often at least one contestant exerting zero effort in equilibrium (Hirshleifer, 1989; Baik, 1997; Che and Gale, 2000). This might be avoided by considering either more general cost functions of effort, like in the previous subsection, or considering a more general prize structure, like in this subsection. In these two cases the resultant CSF is not linear and with sufficient curvature of the function relating costs or prize to effort, an interior equilibrium occurs. Details are left to the reader.

\section{Other Approaches}

Suppose that the prize is the right to supply a certain good (e.g. the Olympic Games) and that the quality of this good is positively related to the effort made by the winner. Let $f_{i}\left(G_{i}\right)$ be the quality of the prize if agent $\mathrm{i}$ wins the contest. Then, expected quality, identified with the utility of the planner is $W(\boldsymbol{p}, \boldsymbol{G})=\sum_{i=i}^{n} p_{i} f_{i}(\boldsymbol{G})$. The planner's problem is an instance of a linear programming problem. Therefore, optimal points assign zero win probability to all but one contestant (or there are multiple optimal points and the planner is indifferent between several adjacent corner point solutions and convex combinations of them). This implies that imperfectly discriminating CSFs cannot be rationalized. ${ }^{12}$ This is illustrated by the following example.

Example 1 Incumbency advantage (Konrad (2002)). Let the contest be winner-pay and $n=2$. Assume $f_{1}\left(G_{1}\right)=G_{1}$ and $f_{2}\left(G_{2}\right)=b G_{2}-a$, where $b \in(0,1]$ and $a \geq 0$. When $a=0$ and $b=1$,

\footnotetext{
${ }^{11}$ The assumption that the value of the prize depends on efforts relates our paper to Ray and Ueda (1996) who analyze a situation in which joint output is shared following a social welfare function but effort decisions are taken on a selfish basis. As in our setting, commitment to a given sharing rule is not possible. Contrary to us, the results in Ray and Ueda are very sensible to the type of social welfare function assumed. In our setting both a Rawlsian and an Utilitarian planner yield difference-form CSFs. This difference between the results of Ray and Ueda and ours is due to the fact that their result is no longer true when individual utility has a separable linear representation in consumption (which it has in our case, see the discussion on pp. 330-331 of their paper).

${ }^{12}$ For the same reason it is also difficult to derive imperfectly discriminating CSFs using expected utility theory when the contest is all-pay. Suppose the organizer obtains $f_{i}(\boldsymbol{G})=\sum_{j=1}^{n} G_{j}$ no matter which contestant wins, then the organizer's payoffs do not depend on the assignment rule. The organizer is, thus, indifferent between CSFs and any rule including imperfectly discriminating ones can be rationalized in mixed-strategies. But notice that $f_{i}(\boldsymbol{G})$ does not need to be the same for all $i$, in which case imperfectly discriminating CSFs cannot be generated. To see this assume that $\boldsymbol{p}(\boldsymbol{G})$ is imperfectly discriminating and $\boldsymbol{p}(\boldsymbol{G})$ can be rationalized in mixedstrategies. Then there exist $\hat{\boldsymbol{G}} \in \mathbb{R}_{++}^{n}$ and $i, j \in N$ such that $f_{i}(\hat{\boldsymbol{G}})>f_{j}(\hat{\boldsymbol{G}})$ and maximizing $W(\boldsymbol{p}, \hat{\boldsymbol{G}})$ requires $f_{j}(\hat{\boldsymbol{G}})=0$. This contradicts that $\boldsymbol{p}(\boldsymbol{G})$ is imperfectly discriminating.
} 
this function rationalizes the standard first-price (sealed bid) auction in mixed-strategies. For other parameter values it rationalizes a biased version of it.

In order to derive imperfectly discriminating CSFs one needs thus to assume some form of non-expected utility theory. See Machina (2004), and especially the table on p. 4, for a survey of these theories.

Here we only consider prospect theory. A prospect is an utility level $f_{i}\left(G_{i}\right)$, which we identify with the quality of the prize, and a probability $p_{i}$. In prospect theory the former is transformed through a value function, while the latter enters the utility through a weighting function. Both functions are assumed to be power functions. ${ }^{13}$

More precisely, consider the following functional form for $W(\boldsymbol{p}, \boldsymbol{G})$ which corresponds to a special case of the class postulated by Kahneman and Tversky (1979, p. 276) for regular prospects, namely ${ }^{14}$

$$
W(\boldsymbol{p}, \boldsymbol{G})=\sum_{i=1}^{n} p_{i}^{\alpha} f_{i}\left(G_{i}\right)^{1-\alpha}, 1>\alpha>0 .
$$

Notice that (20) is the sum of $n$ terms - one associated to each contestant. In each such term both components of a prospect are combined in a Cobb-Douglas way under the assumption of constant returns to scale. Individual utility functions of the Cobb-Douglas form have recently been used in Liu and Wang (2010) in order to analyze sealed bid auctions.

Finally, all these contestants' specific Cobb-Douglas functions are aggregated in an additive way. This reflects that contestants are perfect substitutes from the contest organizer's point of view and implies that the marginal product of a contestant's effort does not depend on the effort of others. Notice lastly that except for the contestants' specific $f_{i}(\cdot)$ 's, $(20)$ is symmetric in contestants. For example, the exponents $\alpha$ and $1-\alpha$, which measure the elasticity of the contest organizer's payoff with respect to effort and win probability, take the same value for every participant. Moreover, there is no contestants' specific scaling parameter. Now we have the following result:

Proposition 7 The contest success function of the form (2) is the unique that can be rationalized in mixed-strategies by a function fulfilling (20).

Proof. Let us maximize $W(\boldsymbol{p}, \boldsymbol{G}), \boldsymbol{p} \in S$. Since $W(\boldsymbol{p}, \boldsymbol{G})$ is continuous on $\boldsymbol{p}$ and $S$ is compact, a maximum exists. Since $W(\boldsymbol{p}, \boldsymbol{G})$ is strictly concave on $\boldsymbol{p}$ and $S$ is convex the maximum is unique. Consider the first order conditions of the maximization with respect to $\boldsymbol{p}$

$$
\alpha p_{i}^{\alpha-1} f_{i}\left(G_{i}\right)^{1-\alpha}-\lambda=0, i=1,2, \ldots, n .
$$

\footnotetext{
${ }^{13}$ Value functions in the form of power functions are often used. For a discussion and axiomatic analysis of the so-called probability weighting functions, including the power function employed in (20), see Prelec (1998). Of course, an important difference between our setting and standard applications of prospect theory is that here win probabilities are an object of choice of the decider.

${ }^{14}$ Although Kahneman and Tversky consider $n=2$, they consider the extension to more outcomes "straightforward" (p. 288).
} 
Clearly, the maximum is interior because if $p_{i} \rightarrow 0$, the left hand side of the above equation goes to infinity. The above equations imply that

$$
p_{i}^{\alpha-1} f_{i}\left(G_{i}\right)^{1-\alpha}=p_{j}^{\alpha-1} f_{j}\left(G_{j}\right)^{1-\alpha}, i, j=1,2, \ldots, n .
$$

Which yield

$$
p_{i}=\frac{f_{i}\left(G_{i}\right) p_{n}}{f_{n}\left(G_{n}\right)}, i=1,2, \ldots, n .
$$

Substituting these equations in the simplex we obtain that

$$
p_{n}=\frac{f_{n}\left(G_{n}\right)}{\sum_{j=1}^{n} f_{j}\left(G_{j}\right)} .
$$

Substituting this equation in the previous one we obtain (2).

Notice that besides prospect theory there might be other interpretations of (20) that depending on the context might be meaningful. For instance, by choosing $f_{i}\left(G_{i}\right)=\left(G_{i}\right)^{\frac{\alpha}{1-\alpha}}$ and by applying to $(20)$ the increasing transformation $V(\boldsymbol{p}, \boldsymbol{G})=(W(\boldsymbol{p}, \boldsymbol{G}))^{1 / \alpha}$, we obtain the CES utility function with the form

$$
V(\boldsymbol{p}, \boldsymbol{G})=\left(\sum_{i=1}^{n}\left(p_{i} G_{i}\right)^{\alpha}\right)^{1 / \alpha} \text { with } \alpha=1-\frac{1}{\sigma},
$$

where $\sigma$ is the elasticity of substitution which is usually assumed to be strictly larger than one. Since the maximizer is not affected, we obtain $p_{i}=\left(G_{i}\right)^{\sigma-1} /\left(\sum_{j=1}^{n}\left(G_{j}\right)^{\sigma-1}\right)$, which allows to explain the exponents in this CSF as the elasticity of substitution of the decider. ${ }^{15}$ In another example one might proceed similarly and choose $f_{i}\left(G_{i}\right)=\left(V_{i}-G_{i}\right)^{\frac{\alpha}{1-\alpha}}$. This corresponds to (4) under the assumption of a winner-pay contest with $\alpha=1-r$ and yields $p_{i}=\left(V_{i}-G_{i}\right)^{\frac{1-r}{r}} /\left(\sum_{j=1}^{n}\left(V_{i}-G_{i}\right)^{\frac{1-r}{r}}\right)$.

\section{Concluding Remarks}

We have shown that contest success functions can be viewed as optimal choices of a contest organizer, given efforts of contestants. This implies that it is not necessary to assume that a contest organizer is able to commit to employ a given contest success function. This approach does not rely on uncertainty or reputation effects. We have shown that our approach works both for all-pay and winner-pay contests. It can also motivate both interpretations of contest

\footnotetext{
${ }^{15}$ Under the assumption that the CSF assigns shares of the prize, the CES function indicates a taste for variety. A politician might have a taste for variety when the lobbying efforts are based on different expertise e.g. when they are used to draft legislation. One interpretation of equation (21) is then that lobbies offer to invest effort $G_{i}$ in drafting a complete bill and that the decider prefers to have parts of the legislation drafted by different lobbies.
} 
success functions: win probabilities and shares of the prize. Our exercise yields contest success functions that were already 'popular' providing new support for them.

Notice that the our approach is very related to, at least, two classical problems.

The first classical problem is the equivalence between revealed preference and utility maximizing choices. For a long time our profession struggled to find a condition under which these two approaches are equivalent. The final solution was obtained by Kihlstrom, Mas-Colell and Sonnenschein (1976). In our case we show the equivalence between contest success functions whose axiomatic properties were known and the choices made by a maximizing agent (the planner).

The second classical problem is the equivalence between market equilibrium and welfare optimum, i.e. the so-called two fundamental theorems of welfare economics. These theorems assert the equivalence between market equilibrium and allocations obtained by a benevolent and well-informed planner, see Mas-Colell (1985 and 1986). In our case, we find that some contest success functions maximize expected social welfare. As in the case of the two fundamental theorems of welfare economics we do not mean that the planner actually exists. The planner is just a surrogate of what the system achieves on its own forces. Thus, in our case, contest success functions can be determined by the pure form of conflict, random elements, etc. What our results say is that these contest success functions are like if they were chosen by a planner (with a visible hand) in order to maximize social welfare.

An interesting implication of our analysis is that contest success functions might depend on the valuations of contestants. This is reasonable when the decider takes into account the welfare effects of her decision on contestants which in a contest model depend on valuations. In this sense our analysis bridges the contest literature and the recent literature on political economy in which the decider takes into account the welfare of rent-seekers (see Grossman and Helpman, 2001).

By postulating reasonable aims for the decider we have derived commonly used contest success functions and new ones with a mathematical structure similar to popular ones. Future research might postulate further payoff functions for the contest organizer and investigate the consequences for the arising contest success functions.

In contrast, in the contest literature many studies suppose that the contest organizer is only interested in maximizing total expected effort. This seems to be a first approximation to what contest organizers care about in many applications. In the present paper we have postulated alternative aims for the contest organizer and shown how these aims translate into contest success functions. We hope that our approach opens the door to taking into account further variables influencing contest organizers.

\section{References}

[1] Baik, Kyung Hwan (1998), "Difference-Form Contest Success Functions and Effort Levels in Contests," European Journal of Political Economy 14, 685-701. 
[2] Blavatskyy, Pavlo R. (2010), "Contest success function with the possibility of a draw: axiomatization," Journal of Mathematical Economics 46, 267-276.

[3] Bossert, Walter and John A. Weymark (2004). Utility in social choice. In S. Barberà, P. Hammond and C. Seidl (Eds.), Handbook of Utility Theory, vol. 2: Extensions. Kluwer, Dordrecht, 1099-1177.

[4] Che, Yeon-Koo and Ian Gale (2000), "Difference-Form Contests and the Robustness of All-Pay Auctions," Games and Economic Behavior 30, 22-43.

[5] Cheng, Leonard K. and Min Zhu (1995), "Mixed-Strategy Nash Equilibrium Based upon Expected Utility and Quadratic Utility," Games and Economic Behavior 9 (2), 139-150.

[6] Corchón, Luis and Matthias Dahm (2010), "Foundations for Contest Success Functions," Economic Theory 43, 81-98.

[7] Dasgupta, Ani and Kofi O. Nti (1998), "Designing Optimal Contests," European Journal of Political Economy 14, 587-603.

[8] Epstein, Gil S. and Shmuel Nitzan (2006), "The Politics of Randomness," Social Choice and Welfare 27, 423-433.

[9] Grossman Gene M. and Elhanan Helpman (2001) Special interest politics. MIT Press, Cambridge.

[10] Grossman, Gene M. and Elhanan Helpman (1994) "Protection for sale," American Economic Review, 84 (4): 833-850.

[11] Jia, Hao (2008), "A Stochastic Derivation of Contest Success Functions," Public Choice 135, $125-130$.

[12] Kahneman, Daniel and Amos Tversky (1979), "Prospect theory: An analysis of decisions under risk," Econometrica, 47, 313-327.

[13] Kihlstrom, Richard; Mas-Colell, Andreu and Hugo Sonnenschein (1976), "The Demand Theory of the Weak Axiom of Revealed Preference," Econometrica, 44 (5), 971-978.

[14] Konrad, Kai A. (2009). Strategy and Dynamics in Contests. Oxford University Press.

[15] Konrad, Kai A. (2002), "Investment in the absence of property rights: the role of incumbency advantages," European Economic Review 46, 1521-1537.

[16] Hirshleifer, Jack (1989), "Conflict and Rent-Seeking Success Functions: Ratio vs. Difference Models of Relative Success," Public Choice 63, 101-12.

[17] Liu, Shulin and Mingxi Wang (2010), "Sealed-bid auctions based on Cobb-Douglas utility function," Economics Letters 107 (1), 1-3. 
[18] Machina, Mark J. (2004), "Non-Expected Utility Theory" in Encyclopedia of Actuarial Science, ed. by J. Teugels and B. Sundt, John Wiley \& Sons.

[19] Mas-Colell, Andreu (1985). Pareto Optima and Equilibria: The Finite Dimensional Case. In C. Aliprantis, O. Burkinshaw and N. Rothman, (Eds.), Advances in Equilibrium Theory, Lecture Notes in Economics, No. 244, Springer-Verlag.

[20] Mas-Colell, Andreu (1986). Valuation Equilibrium and Pareto Optimum Revisited. In A. Mas-Colell and W. Hildenbrand, eds, Chapter 17 in Advances in Mathematical Economics, North-Holland, 1986.

[21] Münster, Johannes (2009), "Group Contest Success Functions," Economic Theory 41 (2), 345-357.

[22] Persson Torsten and Guido Tabellini (2000) Political economics: explaining economic policy. MIT Press, Cambridge.

[23] Prelec, Drazen (1998), "The probability weighting function," Econometrica 66 (3), 497-527.

[24] Rai, Birendra K. and Rajiv Sarin (2009), "Generalized contest success functions," Economic Theory 40 (1), 139-149.

[25] Ray, Debraj and Ueda, Kaoru (1996), "Egalitarianism and Incentives," Journal of Economic Theory $\mathbf{7 1}$ (2), 324-348.

[26] Skaperdas, Stergios (1996), "Contest Success Functions," Economic Theory 7, 283-290.

[27] Skaperdas, Stergios and Li Gan (1995), "Risk aversion in contests", The Economic Journal 105, 951-962.

[28] Skaperdas, Stergios and Samarth Vaidya (2010), "Persuasion as a Contest," Economic Theory forthcoming.

[29] Tullock, Gordon (1980). Efficient rent seeking. In J. Buchanan, R. Tollison, and G. Tullock (Eds.), Toward a theory of the rentseeking society. College Station: Texas A\&M University Press, 97-112.

[30] Wärneryd, Karl (1998), "Distributional conflict and jurisdictional organization," Journal of Public Economics 69, 435-450.

[31] Wärneryd, Karl (2000), "In defense of lawyers: moral hazard as an aid to cooperation", Games and Economic Behavior 33, 145-158.

[32] Yates, Andrew James (2010), "Winner-Pay Contests," Public Choice forthcoming. 